\title{
Laryngeal transplantation in minipigs: vascular, myologic and functional outcomes
}

\author{
M. A. Birchall · P. J. Kingham · P. J. Murison • \\ S. M. Ayling $\cdot$ R. Burt $\cdot$ L. Mitchard $\cdot$ A. Jones $\cdot$ P. Lear $\cdot$ \\ C. R. Stokes $\cdot$ G. Terenghi $\cdot$ M. Bailey $\cdot$ P. Macchiarini
}

Received: 9 April 2010 / Accepted: 25 July 2010 / Published online: 15 September 2010

(C) Springer-Verlag 2010

\begin{abstract}
There is no effective way of replacing all the functions of the larynx in those requiring laryngectomy. Regenerative medicine offers promise, but cannot presently deliver implants with functioning neuromuscular units. A single well-documented laryngeal transplant in man was a qualified success, but more information is required before clinical trials may be proposed. We studied the early response of the larynx to laryngeal transplantation between 17 pairs of $\mathrm{NIH}$ minipigs full matched at the MHC2 locus. Following iterative technical improvements, pigs had good swallowing and a patent airway at 1 week. No significant changes in mucosal
\end{abstract}

This work was done at the Universities of Bristol and Manchester.

M. A. Birchall

Department of Clinical Sciences at South Bristol,

Bristol, UK

M. A. Birchall

Royal United Hospital Bath MHS Trust, Bath, UK

P. J. Kingham $\cdot$ G. Terenghi

Blond McIndoe Laboratories,

Tissue Injury and Repair Research Group,

School of Clinical and Laboratory Sciences,

University of Manchester, Manchester, UK

P. J. Murison · A. Jones

Division of Companion Animals Science,

Department of Clinical Veterinary Sciences,

Faculty of Biomedical Sciences,

University of Bristol, Bristol, UK

S. M. Ayling · R. Burt · L. Mitchard · C. R. Stokes · M. Bailey Division of Veterinary Pathology, Infection and Immunity, Department of Clinical Veterinary Sciences,

Faculty of Biomedical Sciences,

University of Bristol, Bristol, UK blood flux were observed compared with pre-operative measurements. Changes in muscle morphology and fibre phenotype were observed in transplant muscles retrieved after 7 days: the levels of fast and slow myosin heavy chain (MyHC) protein were reduced and embryonic MyHC was up regulated consistent with denervation induced atrophy. At 1 week laryngeal transplantation can result in good swallowing, and is not associated with clinical evidence of ischemia-reperfusion injury in MHC-matched pigs.

Keywords Larynx $\cdot$ Transplantation $\cdot$ Pig

P. Lear

Department of Surgery,

North Bristol NHS Trust, Bristol, UK

M. A. Birchall $(\bowtie) \cdot$ P. Macchiarini

UCL Ear Institute,

Royal National Throat Nose and Ear Hospital,

University College London, 330-332,

Gray's Inn Road, London WC1X 8EE, UK

e-mail: m.birchall@ucl.ac.uk

P. Macchiarini

Department of Cardiothoracic Surgery,

Hospital Careggi, Florence, Italy

Present Address:

M. A. Birchall

Department of Integrative Medical Biology,

Section of Anatomy, Umeå University,

SE-901 87 Umeå, Sweden 


\section{Introduction}

In 2008 , there were 12,250 new cases of laryngeal cancer in USA [1], and a significant proportion of these will ultimately require laryngectomy for treatment of primary or recurrent disease. In addition, there is a smaller, but severely impaired group of patients with non-functioning larynges due to benign disease and trauma [2]. Recent chemoradiation protocols have coincided with modest survival gains for advanced laryngeal cancer, although sometimes at the expense of function; laryngectomy remains a mainstay of treatment. Despite our recent success in clinical airway tissue engineering [3], there is no practical way of substituting the complex functions of the larynx using free tissue transfer, prosthetics or present regenerative medicine techniques. Hence, laryngeal transplantation remains an attractive solution to the considerable quality-of-life impairment faced by laryngectomees $[4,5]$.

The viability of laryngeal transplantation was confirmed by the single well-documented human laryngeal transplant recipient $[6,7]$ who continues to thrive a decade after transplantation with excellent speech and normal swallowing [7]. There is also a report of successes with tracheal transplantation in Colombian patients [8]. These cases demonstrate what may be possible for such patients, but the lack of detailed information on any other than the 1998 patient indicates that more pre-clinical work is needed before laryngeal transplant is a realistic option for most patients.

In two studies of patients' views, the main concern was the level of immunosuppression required by transplant recipients, especially important as the target population includes those with cancer $[9,10]$. However, most people would accept a moderate level of risk in return for substantial gains in quality of life [10]. Before examining the immunological response to grafting, it is essential to measure the response of grafts to ischemia and reperfusion, as well as to determine the functional results of laryngeal transplantation [11]. If we want to separate these responses from those involved in tissue rejection this needs to be done using MHC-matched donors and recipients.

Previous work with dogs and rats permitted major progress, but also had limitations [12-15]. There is a limited range of immunological probes available for studying mucosal responses in dogs. Rats require buried, heterotopic grafts which make repeated observations and functional assessments impossible [14]. Pigs have genetically welldefined strains with a choice of SLA antigen expression ideal for transplant studies [16], and also have welldescribed neuromuscular $[17,18]$ and mucosal immune anatomy [19].We developed an orthotopic laryngeal transplantation in NIH minipigs [20] which mirrored the clinical setting [6] and have previously reported results from the first $8 \mathrm{~h}$ after revascularisation of donor larynges in recipient pigs [20]. The present paper is a logical extension of our previous work $[18,20]$ and is based on the consensus findings of the World's first symposium on this subject [11].

We aimed to measure the response to transplantation at 1 week. One week is a key milestone as it is the point of functional rehabilitation for human laryngectomees: the point at which sufficient healing of the pharyngeal anastomosis has occurred to permit swallowing and speech rehabilitation commences. A study of pharygeosophageal reconstruction after total larygopharyngectomy[21] found that, on average, patients needed 1.1 days ventilator support and left hospital after 4.7 days. In the 1998 transplant [6] the patient had some speech after 1 week.

This study also permitted the training of a group of clinicians in techniques, including post-operative care and monitoring which will be relevant to planned clinical trials. Here, we present functional, vascular and myologic observations at $48 \mathrm{~h}$ and 1 week after transplantation at which time points we expected to see immune responses to ischaemiareperfusion injury and to the onset of rejection, respectively [22]. The immunological response of the laryngeal mucosa to transplantation is reported separately.

\section{Materials and methods}

Animals

About $34 c c$ NIH minipigs (median $17 \mathrm{~kg}$, range 26-48; IAH, Berkshire, UK) were kept under conditions determined by local and national ethical guidelines. Transplants were female-into-female/female-into-male to avoid a host immune response to Y-chromosome-related antigens. Two weeks' acclimatisation before intervention was included to overcome the observed stress responses in pigs that occur following transportation.

Three days before transplantation a percutaneous endoscopic gastrostomy (PEG, Direct Medical Supplies Ltd, Alton, UK) and femoral dual lumen central line (Vygon, Gloucester, UK) were inserted under general anaesthesia.

Initially, open gastrostomies at the time of surgery were planned to permit feeding whilst protecting a healing pharyngeal anastomosis. However, we found in preliminary studies that the tube could become blocked by consumed substances in the animal stomach and that the abdominal surgery appeared to cause the animals distress. Placement of a PEG 3 days prior to surgery reduced the surgical trauma and permitted pigs to become accustomed to the presence and use of a tube in advance of post-operative care.

Likewise, we found that intravenous catheters and arterial lines placed in the ear or leg were poorly tolerated for more than short periods of time and were difficult and 
distressing to replace. Therefore, at the time of PEG insertion, a dual-lumen central line was placed and provided reliable access for drugs and blood sampling for the following week.

\section{Postoperative care}

To reduce morbidity and mortality, and to improve welfare, we developed peri-operative care protocols, including high dependency care [23]. Novel airway management methods, including a T-tube tracheostomy device (patent pending) and pain scoring systems were developed and are reported separately [23]. Dexamethasone $(0.06 \mathrm{mg} / \mathrm{kg})$ was administered intravenously during anaesthesia. Antibiotics and non-steroidal anti-inflammatory drugs were given postoperatively. Opiate analgesia was used. Details of drugs used are provided in Table 1.

Post-operative feeding used milk (Parnutts Foods Ltd., Lincolnshire, UK.). Basic metabolic requirements were calculated as $2.621 \times$ weight $(\mathrm{kg})^{0.63} \mathrm{MJ} /$ day [24] and four times this amount fed to compensate for the increased metabolic rate associated with recovery.

\section{Transplant experiments}

Seventeen fully-MHC2-matched, non-immmunosuppressed transplants were performed using published techniques $[20,25]$. In brief, donor larynges were isolated via midline incisions and perfused with ice-cold University of Wisconsin solution (Dupont, Newcastle, UK) until efflux ran clear. The time of retrieval was recorded for each operation. At induction and after perfusion, mucosal biopsies were taken. Removed organs were placed in bags of University of Wisconsin solution on ice. During this period of cold ischemia, which mimics the time that may be involved in transporting donated organs between hospitals, the operating theatre was prepared for the recipient. Following general anaesthesia and endotracheal intubation, larynges were removed from recipient pigs using smallfield laryngectomy. Ventilation was swapped to a T-tube tracheotomy to facilitate anaesthesia, post-operative care and to stent the anastomosis. Implantation used side-side anastomoses of superior vena cava into recipient right jugular venous confluence and right innominate artery into recipient right common carotid. No attempt was made to repair nerves since the primary aim was to study/perfect the transplant surgery and no functional recovery would occur by 1 week in any case. We have described nerve repair elsewhere $[18,26]$. Mucosal biopsies were taken on reperfusion, which marked the end of cold ischaemia.

Recipients were anaesthetised at $48 \mathrm{~h}$ and grafts inspected via a $0^{0}$ Hopkins rod telescopes (Karl Storz, Berkshire, UK). Biopsies and laser Doppler readings were taken. Recipients underwent a second endoscopy at 7 days, following which they were killed (barbiturate). Grafts were photographed and mucosa and muscle sampled.

\section{Mucosal perfusion}

In order to assess graft perfusion at the capillary level, important since major vessel patency may not correlate with peripheral perfusion in a denervated organ, mucosal blood flux was measured midway along the right false vocal cord using laser Doppler (Moor Instruments, Devon, UK) at 0 (donor prior to organ recovery), 2 and 7 days [27]. After establishing a steady reading, $30 \mathrm{~s}$ recordings were made.

Functional results

Our T-tube device permits airflow through both tracheotomy and larynx. At 1 week, following occlusion of the tracheotomy limb, the ability of the pigs to produce a grunt and flow of air through the nose was assessed. Videofluoroscopy at 1 week assessed swallowing.

Table 1 Drugs, and their doses, used peri-operatively and during the transplant operations

\begin{tabular}{|c|c|c|c|c|}
\hline Drug & Type & Dose & Duration & Source \\
\hline Cefuroxime & Antibiotic & $10 \mathrm{mg} / \mathrm{kg}$, IV 8 hourly & 7 days & GSK, Uxbridge, UK \\
\hline Metronidazole & Antibiotic & $10 \mathrm{mg} / \mathrm{kg}$, oral (via PEG) 12 hourly & 7 days & Hawgreen, Hitchin, UK \\
\hline Dexamaethasone & Steroid & $0.06 \mathrm{mg} / \mathrm{kg}, \mathrm{IV}$ & $\begin{array}{l}\text { During } \\
\text { anaesthesia }\end{array}$ & $\begin{array}{l}\text { Intervet UK Ltd, Milton Keynes, } \\
\text { Bucks UK }\end{array}$ \\
\hline Morphine & Analgesic & $\begin{array}{l}0.2 \mathrm{mg} / \mathrm{kg} \text { every } 4 \mathrm{~h} \text { initially, } \\
\text { then titrated using the pain scale }\end{array}$ & 7 days & $\begin{array}{l}\text { Martindale Pharmaceuticals Ltd, } \\
\text { Romford, Essex }\end{array}$ \\
\hline Ketoprofen $(10 \%)$ & $\begin{array}{l}\text { Non-steroidal } \\
\text { anti-inflammatory }\end{array}$ & $3 \mathrm{mg} / \mathrm{kg}$ & 7 days & $\begin{array}{l}\text { Merial Animal Health, } \\
\text { Harlow Essex UK }\end{array}$ \\
\hline Or meloxicam $(20 \mathrm{mg} / \mathrm{ml})$ & & $0.4 \mathrm{mg} / \mathrm{kg}$ & 7 days & $\begin{array}{l}\text { Boehringer Ingelheim Ltd, } \\
\text { Bracknell, Berkshire, UK }\end{array}$ \\
\hline
\end{tabular}


Myology: immunofluorescence histochemistry

OCT embedded frozen biopsies of laryngeal muscles were prepared as previously described [18]. Briefly, frozen $15 \mu \mathrm{m}$ transverse sections of posterior cricoarytenoid (PCA) muscle and thyroarytenoid (TA) muscles from control and transplant animals were incubated with primary monoclonal myosin heavy chain (MyHC) antibodies described for western blot analysis below, together with rabbit anti-laminin antibody (Sigma) to highlight individual muscle fibres. Primary antibody was detected using secondary antibodies coupled to CY3 or FITC, respectively, and the sections were then viewed using a fluorescence microscope (BX60; Olympus, Tokyo, Japan).

\section{Myology: western blotting}

Sections of muscle were homogenised in lysis buffer containing $100 \mathrm{mM}$ PIPES, $5 \mathrm{mM} \mathrm{MgCl}, 20 \%$ (v/v) glycerol, $0.5 \%$ (v/v) Triton X-100, 5 mM EGTA and protease inhibitors (Sigma, UK). Ten $\mu$ g protein per sample was denatured at $95^{\circ} \mathrm{C}$ for $5 \mathrm{~min}$ and resolved at $120 \mathrm{~V}$ on $10 \%$ SDSPAGE gels. Following transfer to nitrocellulose, membranes were blocked for $1 \mathrm{~h}$ in $5 \%(\mathrm{w} / \mathrm{v})$ non-fat dry milk in TBS-Tween, and then incubated overnight at $4{ }^{\circ} \mathrm{C}$ with either monoclonal fast WB-MHCf (Leica Biosystems, Newcastle, UK; 1:2,000) or slow WB-MHCs (Leica Biosystems; 1:1,000) or embryonic F1.652 MyHC (Alexis Corporation, Nottingham, UK; 1:50) antibodies. Following six 5-min TBS-Tween washes, membranes were incubated for $1 \mathrm{~h}$ with HRP-conjugated secondary antibodies (goat anti-mouse 1:1,000; Cell Signalling Technology, USA). Membranes were washed and treated with chemiluminescent substrate (Amersham, UK) for $1 \mathrm{~min}$. The blots were then exposed (1-10 $\mathrm{min})$ to light-sensitive film, scanned and analysed (Scion Image, Scion Corporation, Maryland, USA).

Statistical methods

Western blotting densitometry was analysed using Kruskal-Wallis 1-way ANOVA.

Laser-Doppler results were analysed using analysis of variance (SPSS 12).

\section{Results}

Transplant operations

Median time for recovery and implantation were $150 \mathrm{~min}$ (range $108-210 ; n=17$ ) and $240 \min (165-480)$, respectively; median cold ischemia time was 340 min (300-540).
There was no difference in recovery, cold ischemia or graft implantation time between successful and unsuccessful operations (Fig. 1). Iterative improvements in technique over the first few cases included changing to a dedicated T-tube tracheotomy device with inner tube, and displacing the tracheotomy away from the site of anastomosis to reduce venous compression.

Graft survival, animal mortality and morbidity

Nine grafts were viable, and one more was borderline viable, to the point of censoring. Thus, eight animals were killed prematurely due to graft failure. In addition, 2 more pigs were killed due to airway obstruction, despite having viable grafts. This problem was overcome by developing the dedicated tube above. Thus, 7/17 animals survived with viable grafts at 7 days (Table 2).

In the eight experiments where graft failure occurred, this was apparent by $48 \mathrm{~h}$. Venous obstruction caused four grafts to fail, a problem later avoided by altering the site of the tracheotomy; one failed to perfuse on table due to inadequate heparinization; two failed to perfuse for unknown reasons (Table 3). The healthy grafts were deep pink though edematous at $48 \mathrm{~h}$ (Fig. 2a, b); the edema subsided by 7 days, leaving a paler graft with normal morphology (Fig. 2c, d). At euthanasia, edema remained in the muscular compartment in two pigs (Fig. 2e, f).

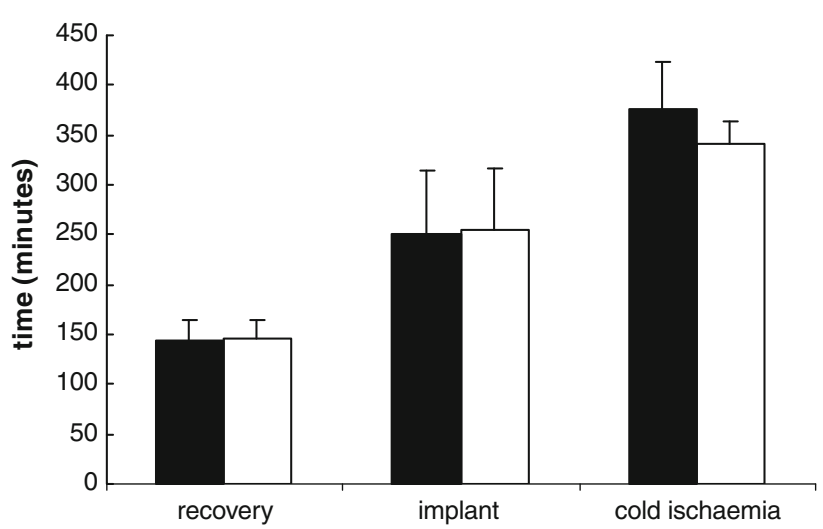

Fig. 1 Mean time for recovery, implantation and cold ischaemia for successful and unsuccessful laryngeal transplants. Figure shows mean with $95 \%$ confidence limits. Filled square successful; opened square unsuccessful

Table 2 Graft survival, animal mortality and morbidity

\begin{tabular}{ll}
\hline Total number of experiments & 17 \\
Viable grafts & 9 or 10 \\
Recipient alive at $48 \mathrm{~h}$ & 13 \\
Recipient alive at 7 days & 7 \\
\hline
\end{tabular}


Table 3 Causes of premature death

\begin{tabular}{llll}
\hline & Cause & Number & Solution \\
\hline Viable graft & Blocked tracheostomy tube & 1 & Developed special T-tube, and protocol for airway cleaning \\
& Lost tracheostomy tube & 1 & \\
Other airway problems & 1 & 4 & $\begin{array}{c}\text { Enlist help of vascular surgeon, displace the tracheotomy } \\
\text { away from the site of anastomosis to reduce venous compression }\end{array}$ \\
& Venous occlusion & & Ensure dose correctly weight adjusted \\
& Heparinisation & 1 & \\
Prolapsing fat/debris airway & 2 & \\
& problems/?dead graft & 10 & \\
\end{tabular}

Fig. 2 Endoscopic appearance of (a), (b) laryngeal transplants at $48 \mathrm{~h}$ and (c), (d) at 7 days. At $48 \mathrm{~h}$, the larynx is highly oedematous, whilst a patent lumen and visible vocal cords reappear by 1 week. Revascularised laryngeal graft 1 week after transplantation: (white arrow vascular pedicle; black arrow vocal cords) on removal (e) and sagittally split posteriorly (arrow healed tracheal anastomosis) (f)
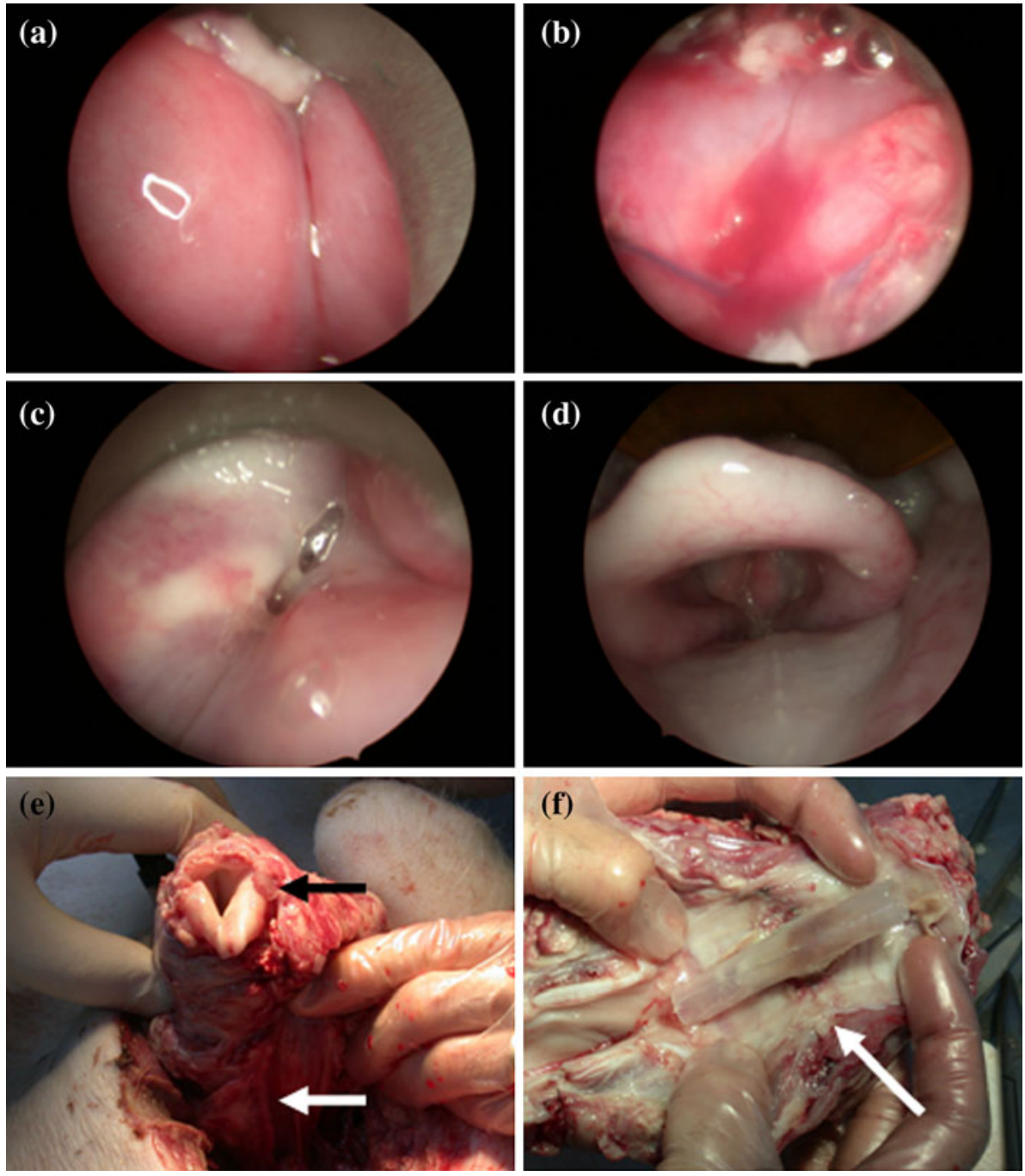

Surviving animals were healthy at 1 week, exhibited normal social behaviour with care staff and experienced negligible weight loss $(0.2 \mathrm{~kg}$, range 1 - gain 0.5$)$. Minor complications encountered were wound infection (one) and wound dehiscence (two). These were easily managed with local measures.
Mucosal perfusion

Laser Doppler flux at the initial measurement was 171 arbitary units (AU) (range 69-418), there was a small increase at $48 \mathrm{~h}$ to $199 \mathrm{AU}$ (range 48-386) and a small decrease at 7 days to $156 \mathrm{AU}$ (56-247). There was no statistical difference 
Fig. 3 a Laser Doppler flux recordings from a normal larynx (top), a successful graft (middle, mean flow 200 arbitrary units) and one which subsequently failed (bottom, mean < 50).

b Blue dye injection into pedicle shows distribution of blood supply to all parts of the larynx. The supraglottis seemed relatively less well perfused, which may contribute to the observed lack of leukocyte changes in this subsite. c Laser Doppler fluxmetry (mean $\pm 95 \%$ confidence limits, arbitrary units) of pig laryngeal graft mucosal blood flux at 0 (donor), 2 and 7 days post- transplantation. Filled square successful; opened square unsuccessful (a)
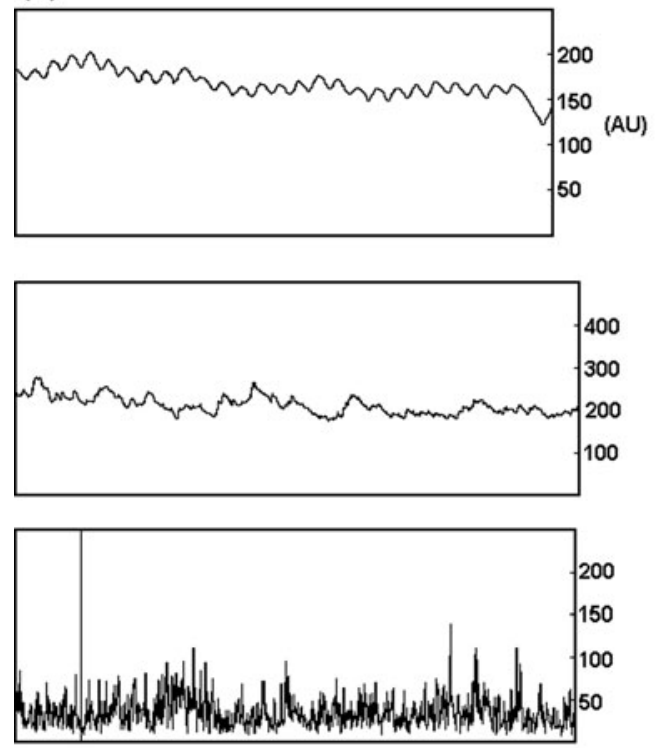

(b)

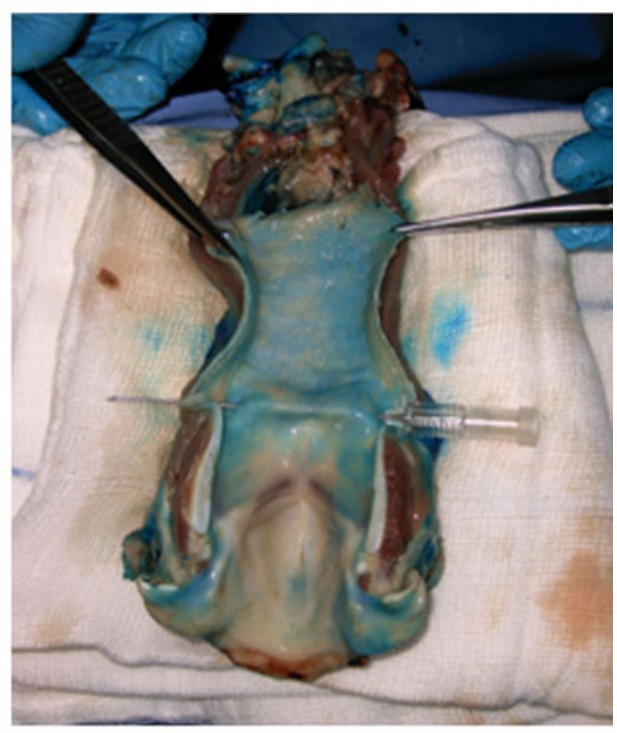

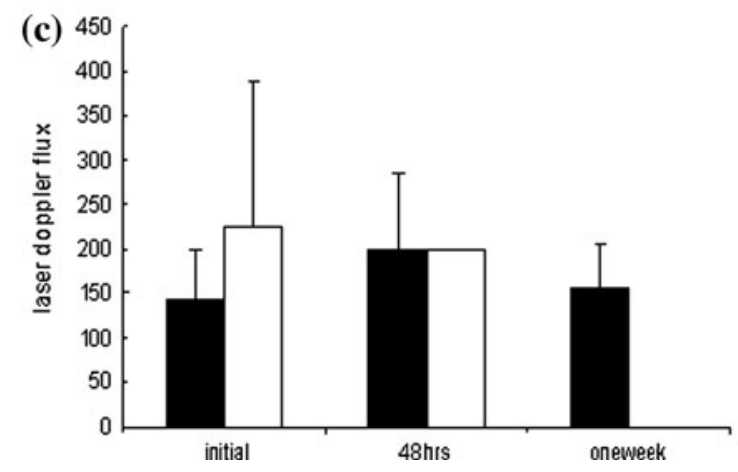

in initial laser Doppler flux between successful and unsuccessful experiments (Fig. 3). Laser Doppler measurements were a useful means of confirming graft failure, with all ischemic larynges exhibiting readings of 50 arbitrary units or less. These values were not included in the analysis.

Functional results

In all seven pigs surviving with healthy grafts to 1 week, occlusion of the vertical limb of the T-tube resulted in passage of some air through the larynx. Audible grunts ('phonation') were produced by five recipient pigs. In no case, however, was airflow sufficient to support nasal breathing at 1 week. Four animals co-operated with videofluoroscopy: three swallowed freely without laryngeal penetration, aspiration or fistula (Fig. 4). One pig experienced minor aspiration which cleared on coughing.

\section{Myology}

Control muscle sections of posterior cricoarytenoid (PCA; Fig. 5a) and thyroarytenoid (TA; Fig. 5b) showed largediameter fibres mainly positive for fast-type myosin heavy

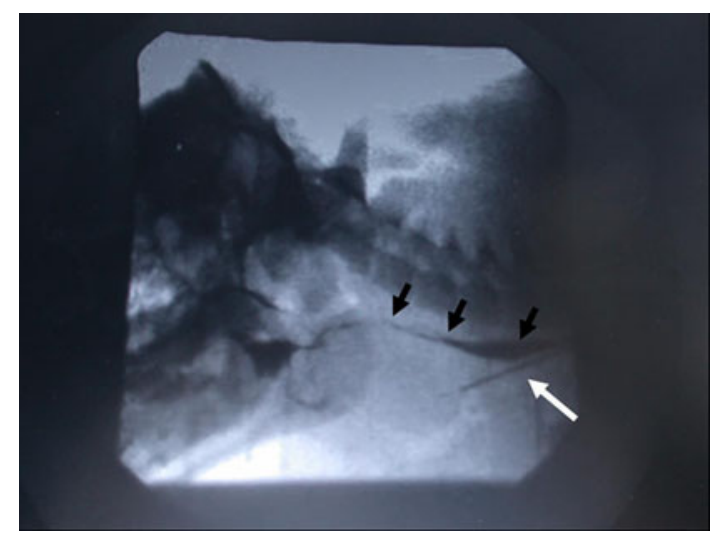

Fig. 4 Barium swallow 1 week post-laryngeal transplantation in a pig. White arrow indicates $t$-tube tracheostomy within the transplanted subglottis. Black arrows show normal swallowing of contrast. There is no aspiration seen

chain (MyHC). There was no expression of embryonic MyHC. However, samples retrieved at 7 days showed large reductions in fibre size, mosaic architecture and immuno-positivity for fast and slow type MyHC (Fig. 5a, b). Embryonic MyHC was expressed in some muscle fibres. Western blotting showed a significant reduction in levels of 
Fig. 5 Morphology of laryngeal muscle fibres. a Posterior cricoarytenoid (PCA) muscle. Transverse sections of control PCA (top row) and transplant PCA (bottom row) muscles stained with laminin antibody (green) and either fast type, slow type or embryonic MyHC protein antibody (red). b Thyroarytenoid (TA) muscle. Transverse sections of control TA (top row) and transplant TA (bottom row) muscles stained with laminin antibody (green) and either fast type, slow type or embryonic MyHC protein antibody (red) (a)

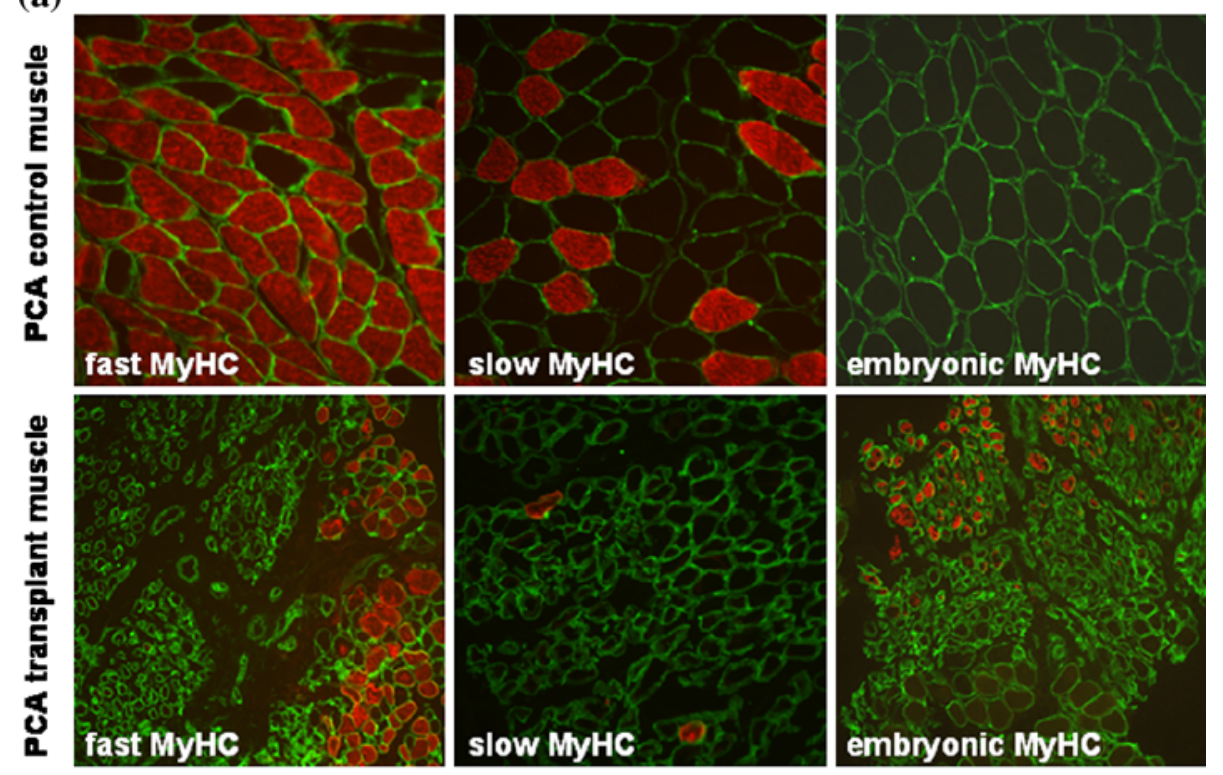

(b)
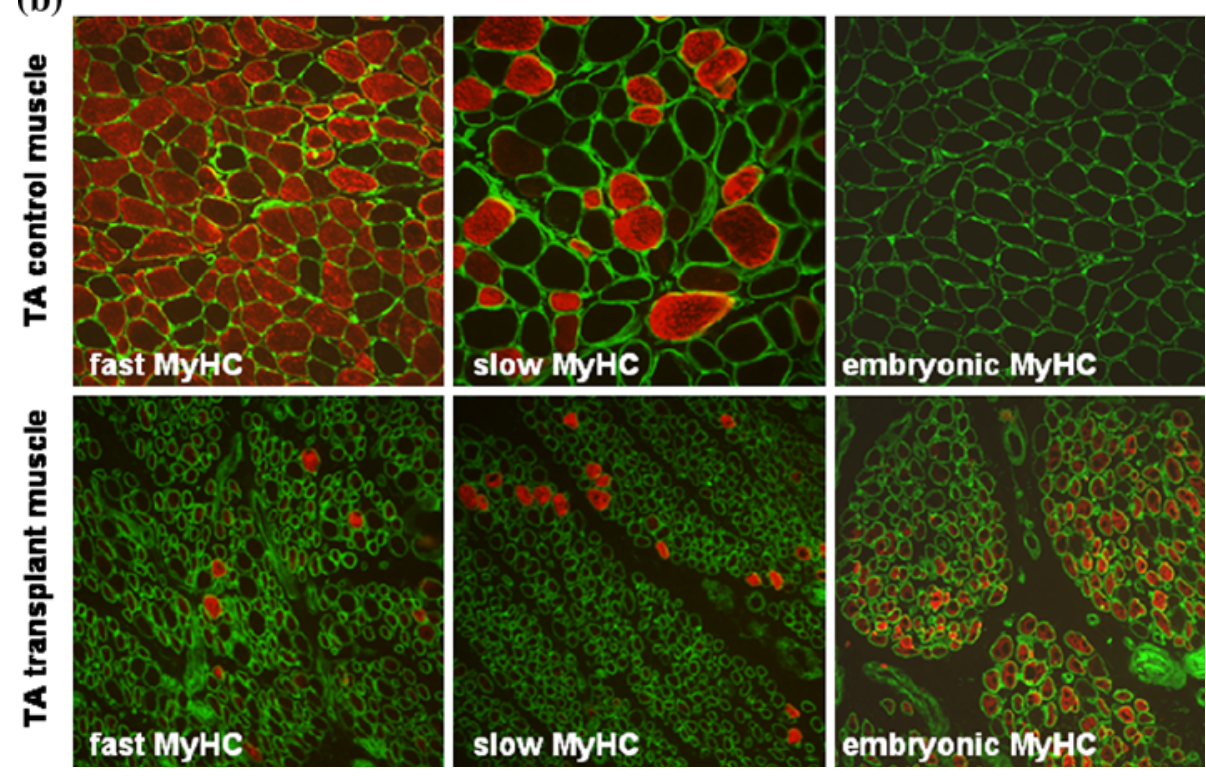

fast and slow MyHC in transplant muscles. Embryonic MyHC was expressed only in transplanted muscle (Fig. 6).

\section{Discussion}

This is the first comprehensive description of the performance, management and early outcomes of laryngeal transplantation in a large animal and provides valuable preclinical data and experience for human trials. Following incremental technical modifications (dedicated T-tube, improved vascular technique, used of PEG tube and central line) good graft and pig survival were achieved in this valuable but labour-intensive model. Mucosal perfusion was sustained and normal by 1 week in surviving animals. Mucosal oedema was transient, leading to some airway patency and normal swallowing at 1 week. Laryngeal muscle morphology and phenotype altered significantly, consistent with denervationinduced atrophy. These data provide important baseline observations for further interventional studies, including unmatched grafts, long-term survival studies and studies of reinnervation.

\section{Graft survival and function}

Operating times compared favourably with those for other complex grafts [28, 29]. High morbidity (>50\%) is common in experimental work involving major surgery in pigs. 
Fig. 6 Changes in myosin heavy chain protein expression in transplanted muscle. a Western blots of lysates were prepared from the PCA and TA muscles from control animals (con) and transplanted (trans) muscle 7 days following surgery. Samples were electrophoresed and transferred to nitrocellulose membranes as described in "Materials and methods". Membranes were probed with antibodies directed against fast, slow, or embryonic type MyHC protein. b Graphs show densitometry data as mean \pm SEM values $(n=5$ animals) $* P<0.05 ; * * P<0.01$; $* * * P<0.001$ significantly different when transplant muscle is compared with control muscle (a)

PCA muscle

TA muscle

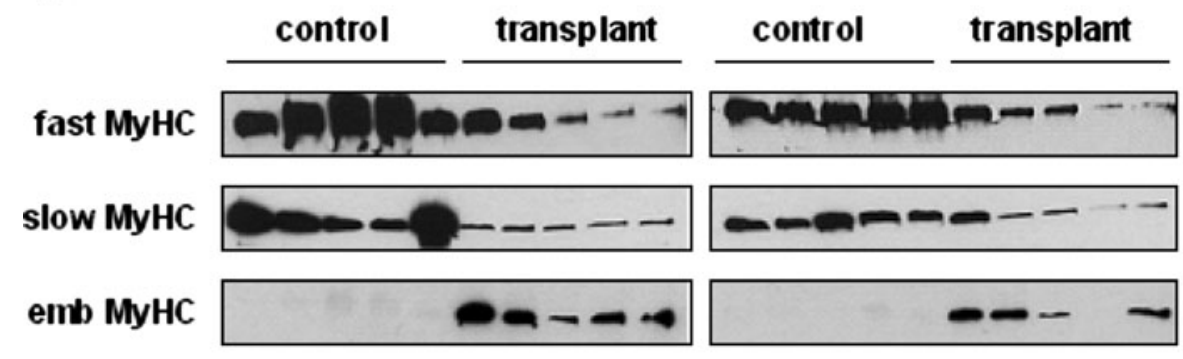

(b)

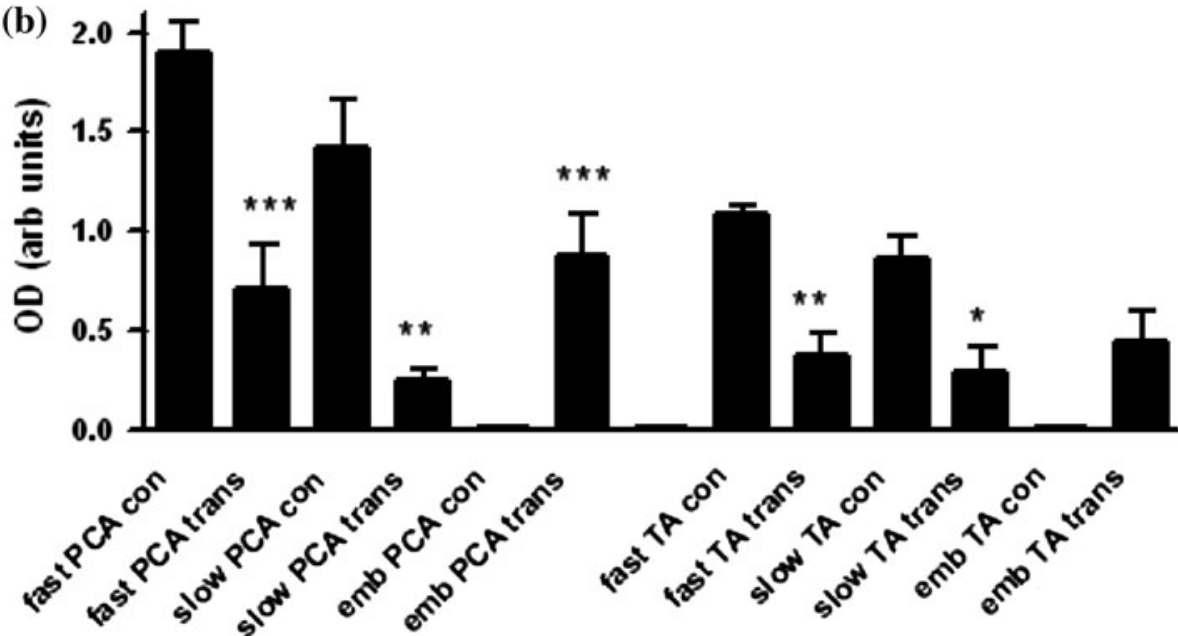

Nevertheless, incremental improvements in peri-operative care and operating technique during the series culminated in $100 \%$ peri-operative survival of the final 12 recipients.

We would hope that any future animal or human trial would have better survival rates. The improvements in surgical technique, such as placement of the graft, would be applied. Human patients would understand the surgical procedure and be more likely to comply with requests; for example, to keep still. In animal trials ethical considerations and licensing rules prevent any further intervention, whereas in a human if it were apparent that the graft had failed the graft could be removed.

The combination of videofluoroscopy, endoscopy, laser Doppler and measurement of airflow through the T-tube gave us confidence that the viable grafts were functional. In general the graft was not oedematous after 1 week; therefore, we think it unlikely that swallowing without aspiration was due to oedema at the superior part of the larynx. The mucosa of one graft remained oedematous at 1 week. The one reported human laryngeal graft exhibited initial oedema which subsided sufficiently to permit speech by 1 week [6], as observed in the surviving pig larynges here. This oedema may represent part of the normal range of responses, or a response to minor antigen mismatches. However, our detailed immunological studies (presented in a separate paper) found no evidence to support the latter.
Mucosal perfusion

We have previously validated the use of laser Doppler fluxmetry as a measure of laryngeal mucosal blood flux in man and pig [27]. Although laser Doppler measurements failed to predict success and failure in the present study, we found them useful in making decisions about the viability of grafts in the early post-operative period and may have clinical utility in this role; further studies would be required.

\section{Myology}

In two of five grafts for which detailed studies were performed, transplanted muscle was macroscopically identical to control muscle taken from the recipient's larynx prior to implantation. In other samples, breakdown of muscle structure, reductions in muscle fibre size and changes in phenotype were apparent, despite no significant changes in appearance or immunohistology of the mucosal compartment in the same larynges. This is most likely to be a consequence of denervation-induced atrophy. In a previous study on primate laryngeal muscles it was shown that denervation produced shrinkage of fast-type fibres within 2 weeks (particularly in the thyroarytenoid) and after 8 weeks all laryngeal muscles showed significant fibrosis [30]. Similarly, in a rat model of recurrent laryngeal nerve 
transection there was an approximate $40 \%$ decrease in muscle fibre size and almost complete loss of synaptophysinpositive nerve terminals [31] 2 weeks after denervation. Even as early as 7 days, denervation induces a significant reduction in muscle weight (which recovers upon reinnervation) and decreased fibre size in rat laryngeal muscles, with a large reduction in type IIB myosin heavy chain protein [32]. This is consistent with our results showing a significant fall in fast-type MyHC protein levels; we have previously shown that type IIB MyHC predominates in pig laryngeal muscle [18]. One study [33] suggests slow type I MyHC is relatively unaffected by denervation, but another indicates that over a longer time-course there is a progressive reduction in expression [34]. We also observed a "reactivation" of embryonic myosin heavy chain protein in transplanted muscles. This suggests to us that the atrophic changes occurring as a result of denervation can trigger a regenerative response mimicking the developmental processes of muscle formation [35]. Indeed, recent studies indicate that compared with limb muscles, laryngeal muscle has an enhanced ability to respond to denervation as the result of a robust addition of myonuclei and myofibre remodelling due to intrinsic activation of muscle satellite cells [36, 37]. Thus, further studies are warranted to determine how functional reinnervation might be achieved following laryngeal transplantation. Lack of sensory innervation at 1 week was probably the cause of the minor aspiration noted in one animal, though its absence in the other four undergoing videofluoroscopy was encouraging.

\section{Conclusion}

This is the first detailed study of the acute vascular and myologic outcomes of laryngeal transplantation in a preclinical model. At 1 week, laryngeal transplants between pigs fully matched at the MHC2 loci are characterised by good function as measured by swallowing and 'phonation'. Laryngeal muscle displays signs of denervation induced atrophy, but the "reactivation" of embryonic MyHC is encouraging, suggesting that the muscle can actively mount a regenerative response after transplantation. The knowledge and skills we have gained from this 1-week study would permit us to progress, subject to licensing and ethical approval, with more confidence to longer term studies, in which the function of the graft can be robustly tested over several weeks or months. These studies provide a firm scientific basis on which to interpret the response to allografting in this robust pre-clinical model.

This study also provides some evidence and in vivo experience and training which would support subsequent clinical trials.
Acknowledgments Numerous research fellows, ENT senior house officers and registrars, $\mathrm{PhD}$ students and veterinary nurses assisted with this project. They all gave of their time freely, including spending many hours performing high dependency care of pigs. Every one of them has played a part in the development of this complex, but ultimately successful model and project. Kind advice was received from many parts of the School of Clinical Veterinary Science, University of Bristol, where Professor A. Waterman-Pearson, Dr. K. Haverson, Dr. C.F. Inman and Mr. C. Chambers were particularly supportive. Funding was provided by Wellcome Trust Clinical Research Leave Fellowship to Martin Birchall (GR061125); some equipment kindly provided by Karl Storz UK, Ltd. This project was approved by the Bristol University Ethical Review Group, and by the UK Home Office (PPL 30/2374).

Conflict of interest There is no conflict of interest.

\section{References}

1. National Cancer Institute, US National Institutes of Health. http:// www.cancer.gov, 2009

2. Nouraei SAR, Nouraei SM, Howard DJ, Sandhu GS (2007) Estimating the population incidence of adult post-intubation laryngotracheal stenosis. Clin Otolaryngol 32(5):407-409

3. Macchiarini P, Jungebluth P, Go T et al (2008) Clinical transplantation of a tissue-engineered airway. Lancet 372(9655):2023-2030

4. Meyer TK, Kuhn JC, Campbell BH, Marbella AM, Myers KB, Layde PM (2004) Speech intelligibility and quality of life in head and neck cancer survivors. Laryngoscope 114(11):1977-1981

5. Fung K, Lyden TH, Lee J et al (2005) Voice and swallowing outcomes of an organ-preservation trial for advanced laryngeal cancer. Int J Radiat Oncol Biol Phys 63(5):1395-1399

6. Strome M, Stein J, Esclamado R et al (2001) Laryngeal transplantation and 40-month follow-up. N Engl J Med 344(22):1676-1679

7. Lorenz RR, Hicks DM, Shields RW Jr, Fritz MA, Strome M (2004) Laryngeal nerve function after total laryngeal transplantation. Otolaryngol Head Neck Surg 131(6):1016-1018

8. Rivera EG, Tintinago LF, Velásquez JC, Páramo HA, Gaviria JD, Ramirez GA (2006) Manejo de la via aérea en trasplante de tráqea. Rev Col Anest 34:75-81

9. Potter CPS, Birchall MA (1998) Laryngectomee's views on laryngeal transplantation. Transplant Int 11(6):433-438

10. Reynolds CC, Martinez SA, Furr A et al (2006) Risk acceptance in laryngeal transplantation. Laryngoscope 116(10):1770-1775

11. Birchall MA, Lorenz RR, Berke GS, Genden EM, Haughey BH, Siemionow M, Strome M (2006) Laryngeal transplantation in 2005: a review. Am J Transplant 6(1):20-26

12. Kevorkian KF, Sercarz JA, Ye M, Kim YM, Hong KH, Berke GS (1997) Extended canine laryngeal preservation for transplantation. Laryngoscope 107(12 Pt 1):1623-1626

13. Strome M, Wu J, Strome S, Brodsky G (1994) A comparison of preservation techniques in a vascularized rat laryngeal transplant model. Laryngoscope 104(6 Pt 1):666-668

14. Haug M 3rd, Dan O, Wimberley S, Fritz M, Lorenz RR, Strome M (2003) Cyclosporine dose, serum trough levels, and allograft preservation in a rat model of laryngeal transplantation. Ann Otol Rhinol Laryngol 112(6):506-510

15. Andrews RJ, Berke GS, Blackwell KE, Jakobsen M, Wang MB, Sercarz JA (2000) Hemilaryngeal transplantation in the canine model: technique and implications. Am J Otolaryngol 21(2):85-91

16. Sachs DH, Leight G, Cone J, Schwarz S, Stuart L, Rosenberg S (1976) Transplantation in minature swine. Transplantation 22(6):559-567 
17. Knight MJ, McDonald SE, Birchall MA (2005) Intrinsic muscles and distribution of the recurrent laryngeal nerve in the pig larynx. Eur Arch Otorhinolaryngol 262(4):281-285

18. Kingham PJ, Birchall MA, Burt R, Jones A, Terenghi G (2005) Reinnervation of laryngeal muscles: a study of changes in myosin heavy chain expression. Muscle Nerve 32(6):761-766

19. Barker E, Haverson K, Stokes CR, Birchall M, Bailey M (2005) The larynx as an immunological organ: immunological architecture in pig as a large animal model. Clin Exp Immunol 143:6-14

20. Barker E, Murison P, Macchiarini P, Jones A, Otto C, Rothkoetter HJ et al (2006) Early immunological changes associated with laryngeal transplantation in a major histocompatibility complex-matched pig model. Clin Exp Immunol 146(3):503-508

21. Yu P, Hanasano MM, Skoracki RJ et al (2010) Pharygoesophageal reconstruction with the anterolateral thigh flap after total laryngopharyngectomy. Cancer 116(7):1718-1724

22. Friedman AD, Dan O, Drazba JA, Lorenz RR, Strome M (2007) Postallograft donor and recipient dendritic cell trafficking in the rat larynx. Laryngoscope 117(9):1615-1621

23. Murison PJ, Jones A, Mitchard L, Burt R, Birchall MA (2009) Development of peri-operative care for pigs undergoing laryngeal transplantation. Laboratory Animals June 17 e-pub

24. Agricultural Research Council. The nutrient requirements of pigs/ technical review by an Agricultural Research Council Working Party. Farnham royal: commonwealth agricultural Bureaux, 1981

25. Birchall MA, Bailey M, Barker EV, Rothkotter HJ, Otto K, Macchiarini P (2002) Model for experimental revascularized laryngeal allotransplantation. Br J Surg 89(11):1470-1475

26. Birchall MA, Idowu B, Murison P et al (2004) Laryngeal abductor muscle reinnervation in a pig model. Acta Otolaryngol 124(7): 839-846
27. Jacob A, Birchall M (2003) Laser Doppler flux-metry as an experimental tool in Laryngology. Eur Arch Otorhinolaryngol 260:308-311

28. Dubernard J-M, Owen E, Herzberg G et al (1999) Human hand allograft: report on first 6 months. Lancet 353(9161):1315-1320

29. Abu-Elmagd K, Reyes J, Bond G et al (2001) Clinical intestinal transplantation: a decade of experience at a single center. Ann Surg 234:404-417

30. Sahgal V, Hast MH (1986) Effect of denervation on primate laryngeal muscles: a morphologic and morphometric study. J Laryngol Otol 100(5):553-560

31. Kumai Y, Ito T, Udaka N, Yumoto E (2006) Effects of a nervemuscle pedicle on the denervated rat thyroarytenoid muscle. Laryngoscope 116(6):1027-1032

32. Wu YZ, Baker MJ, Marie JP, Crumley R, Caiozzo VJ (2004) The plasticity of denervated and reinnervated laryngeal muscle: focus on single-fiber myosin heavy-chain isoform expression. Arch Otolaryngol Head Neck Surg 130(9):1070-1082

33. Shiotani A, Flint PW (1998) Myosin heavy chain composition in rat laryngeal muscles after denervation. Laryngoscope 108(8 Pt 1): $1225-1229$

34. DelGaudio JM, Sciote JJ (1997) Changes in myosin expression in denervated laryngeal muscle. Ann Otol Rhinol Laryngol 106(12): 1076-1081

35. Borisov AB, Dedkov EI, Carlson BM (2000) Interrelations of myogenic response, progressive atrophy of muscle fibers, and cell death in denervated skeletal muscle. Anat Rec 264(2):203-218

36. Shinners MJ, Goding GS, McLoon LK (2006) Effect of recurrent laryngeal nerve section on the laryngeal muscles of adult rabbits. Otolaryngol Head Neck Surg 134(3):413-418

37. McLoon LK, Thorstenson KM, Solomon A, Lewis MP (2007) Myogenic precursor cells in craniofacial muscles. Oral Dis 13(2):134-140 